\title{
Fundamental units in a family of cubic fields
}

\author{
par VEIKKO ENNOLA
}

\begin{abstract}
RÉSUMÉ. Soit $\mathcal{O}$ l'ordre maximal du corps cubique engendré par une racine $\varepsilon$ de l'equation $x^{3}+(\ell-1) x^{2}-\ell x-1=0$, où $\ell \in \mathbb{Z}$, $\ell \geq 3$. Nous prouvons que $\varepsilon, \varepsilon-1$ forment un système fondamental d'unités dans $\mathcal{O}$, si $[\mathcal{O}: \mathbb{Z}[\varepsilon]] \leq \ell / 3$.

ABSTRACT. Let $\mathcal{O}$ be the maximal order of the cubic field generated by a zero $\varepsilon$ of $x^{3}+(\ell-1) x^{2}-\ell x-1$ for $\ell \in \mathbb{Z}, \ell \geq 3$. We prove that $\varepsilon, \varepsilon-1$ is a fundamental pair of units for $\mathcal{O}$, if $[\mathcal{O}: \mathbb{Z}[\varepsilon]] \leq \ell / 3$.
\end{abstract}

\section{Introduction}

Many computational methods in number theory depend on the knowledge of the unit group of an order in an algebraic number field. Especially, several parametrized families of cubic orders with a given fundamental pair of units are known (see, e.g., [3] and papers cited there). However, it seems that the results mostly suffer from the incompleteness that either it is not known whether the units also form a fundamental pair of units for the maximal order of the field (cf. the corrigendum to [4]), or this is achieved by imposing a further restrictive condition. E.g., in the case of a non-Galois cubic field this means in practice that the discriminant of the defining polynomial is assumed to be square-free.

In an earlier paper [2] we gathered together basic arithmetic facts and further results and conjectures about the two families of cubic fields containing exceptional units, the main emphasis laying on the non-abelian family. This is the set $\mathcal{F}=\left\{F_{\ell}\right\}$, where

$$
F_{\ell}=\mathbb{Q}(\varepsilon), \quad \operatorname{Irr}(\varepsilon, \mathbb{Q})=f_{\ell}(x)=x^{3}+(\ell-1) x^{2}-\ell x-1, \quad \ell \geq 3 .
$$

For each $\ell$, we fix $\varepsilon$ somehow among its conjugates in order to get a unique field $F_{\ell}$. Here $\ell \geq 3$ is a natural limitation to avoid duplication and to exclude from the family three fields one of which is cyclic and the other two are not totally real.

E. Thomas [5] proved that $\varepsilon, \varepsilon-1$ is a fundamental pair of units for the order $\mathbb{Z}[\varepsilon]$, and in $[2$, Conjecture 4.1$]$ we conjectured that the same is true 
for the maximal order $\mathcal{O}=\mathcal{O}_{F_{\ell}}$. Using the Voronoi algorithm in the cases when the discriminant $D=D\left(f_{\ell}\right)$ of the polynomial $f_{\ell}(x)$ is divisible by the square of a prime $p \neq 7$, we were able to show that the conjecture is true for $3 \leq \ell \leq 500$.

The main problem in this context is whether a unit of the form $\varepsilon^{a}(\varepsilon-1)^{b}$ is a non-trivial $p$ th power in $F_{\ell}$. For $(a, b)=(2,1)$ or $(1,2)$ we showed in [2] that it is not so if $p=5$, and claimed that the same holds for any prime $p$. We have been able to prove this by means of a very tedious computation, the details of which are uninteresting. As suggested in [2], the crucial question here is a successful choice of the approximation polynomial $q$. The following construction seems to work in all cases: Suppose that $\eta^{p}=\varepsilon^{a}(\varepsilon-1)^{b}$, where $\eta \in F_{\ell}, p$ is an odd prime, and $a$ and $b$ are coprime positive integers less than $p$. Put $\delta=1$, if $a$ is even and $b$ is odd, and $\delta=-1$ otherwise. Take

$$
q=\delta \operatorname{Tr}\left(\eta^{-a-b}\right)+\operatorname{Tr}\left(\eta^{a}\right)+\operatorname{Tr}\left(\eta^{b}\right),
$$

where $T r$ denotes the trace from $F_{\ell}$ to $\mathbb{Q}$. This choice is different from the one in [2], but so far it has worked well in each case investigated.

Let $j$ denote the index $[\mathcal{O}: \mathbb{Z}[\varepsilon]]$. Our purpose is to prove the following result which shows that the conjecture is true if $j$ is not too large:

Theorem. If $j=[\mathcal{O}: \mathbb{Z}[\varepsilon]] \leq \ell / 3$, then $\varepsilon, \varepsilon-1$ is a fundamental pair of units for the maximal order $\mathcal{O}$ of the field $F_{\ell}$.

Using Maple we have computed the prime factorization of $D$ and have verified that, for $3 \leq \ell \leq 10000, D$ has a squared factor $k^{2}$ with $k>\ell / 3$ only in a few cases, and that in these cases $k \equiv 0 \bmod 7, D \not \equiv 0 \bmod 7^{3}$, so that (see Lemma 1) $j$ is a divisor of $k / 7$. One can then check that $j \leq \ell / 3$. Therefore, $\varepsilon, \varepsilon-1$ is a fundamental pair of units for $\mathcal{O}$ if $3 \leq \ell \leq 10000$.

\section{Basic lemmas}

These lemmas are contained in [2], but in order to make the proof of the theorem self-contained, we repeat their proofs shortly here. Note that $j^{2}$ is a divisor of $D$, in fact, $D / j^{2}$ is the discriminant of the field $F_{\ell}$. By $p^{m} \| c$ we mean that $p^{m} \mid c, p^{m+1} \nmid c$.

Lemma 1. (i) If $\ell \not \equiv 2 \bmod 7$, then $7 \nmid D$.

(ii) If $\ell \equiv 2 \bmod 7$, but $\ell \not \equiv 23 \bmod 49$, then $7^{2} \| D, 7 \nmid j$.

(iii) If $\ell \equiv 23 \bmod 49$, then $7^{3}\left\|D, 7^{1}\right\| j$.

Proof. The polynomial discriminant $D$ has the expression

$$
D=\ell^{4}+6 \ell^{3}+7 \ell^{2}-6 \ell-31=\left(\ell^{2}+3 \ell-1\right)^{2}-32 .
$$

Note that every prime divisor of $D$ is $\equiv \pm 1 \bmod 8$. 
It is easy to see that (i) holds, e.g., by direct computation. Suppose therefore, that $\ell \equiv 2 \bmod 7$. Substituting $\ell=2+7 n$ in (1) we get

$$
D \equiv 49\left((2 n+1)^{2}+14\left(n^{2}+n\right)\right) \bmod 7^{4} \text {. }
$$

Hence $7^{3} \| D$ only for $n \equiv 3 \bmod 7$, i.e., $\ell \equiv 23 \bmod 49$, and otherwise $7^{2} \| D$.

For $\ell \not \equiv 23 \bmod 49$,

$$
f_{\ell}(x+2)=x^{3}+(\ell+5) x^{2}+(3 \ell+8) x+2 \ell+3
$$

is an Eisenstein polynomial modulo 7 , so that 7 is fully ramified in $F_{\ell}$, and (ii) follows.

For $\ell \equiv 23 \bmod 49$, (iii) is a consequence of $(\varepsilon-2)^{2} / 7 \in \mathcal{O}$. This fact can be seen in many ways, the most straightforward but perhaps not the cleverest method being to compute the minimal polynomial.

Lemma 2. The ring $\mathcal{O}$ has a $\mathbb{Z}$-basis of the form $1, \varepsilon, \alpha$, where $\alpha=(u+$ $\left.v \varepsilon+\varepsilon^{2}\right) / j$, and the integers $u, v$ are determined by $0 \leq u, v<j$ and

(2) $28 u \equiv-2 \ell^{3}-9 \ell^{2}-11 \ell+11, \quad 28 v \equiv \ell^{3}+\ell^{2}+9 \ell-30 \bmod (7, j) j$.

Proof. We shall use a theorem of Voronoi [1, p. 111, Theorem I]. Put $a=$ $2\left(\ell^{2}+\ell+1\right), b=\ell^{2}-\ell-9$. Since the resultant of $a$ and $b$ with respect to $\ell$ equals 336 and $b$ is odd, the gcd $(a, b)$ is a divisor of 21 .

Firstly, we must show that the simultaneous congruences

$$
f_{\ell}(x) \equiv 0 \quad \bmod k^{3}, \quad f_{\ell}^{\prime}(x) \equiv 0 \quad \bmod k^{2}, \quad \frac{1}{2} f_{\ell}^{\prime \prime}(x) \equiv 0 \quad \bmod k
$$

do not have a common solution for any $k>1$. Suppose the contrary. It follows from the identities

$$
\begin{aligned}
9 f_{\ell}(x)-(3 x+\ell-1) f_{\ell}^{\prime}(x) & =-a x+b \\
-12 f_{\ell}^{\prime}(x)+f_{\ell}^{\prime \prime}(x)^{2} & =2 a
\end{aligned}
$$

that $k^{2} \mid(a, b)$, a contradiction.

Hence $\mathcal{O}$ has an integral basis of the required form, where $u$ and $v$ have to be determined. Put $j^{\prime}=j /(7, j)$, so that $7 \nmid j^{\prime}$. By Voronoi's theorem we have

$$
u \equiv t^{2}+(\ell-1) t-\ell, \quad v \equiv t+\ell-1 \quad \bmod j^{\prime}
$$

where $t$ is a solution of the congruences

$$
f_{\ell}(t) \equiv 0 \bmod j^{2}, \quad f_{\ell}^{\prime}(t) \equiv 0 \quad \bmod j^{\prime}
$$

Further useful identities are

$$
\begin{gathered}
a^{2} f_{\ell}^{\prime}(b / a)=-9 D \\
a\left(3 \ell^{3}+10 \ell^{2}-22 \ell-41\right)+392=(6 \ell-10) D
\end{gathered}
$$


It follows from (7) that $\left(a, j^{\prime}\right)=1$, and then from the equations (3), (6) that $t=b / a$ is a solution of (5). Substituting $t=b / a$ in (4) and using (7) to remove the denominators, we obtain after a short computation the congruences (2) modulo $j^{\prime}$.

Suppose finally that $7 \mid j$, i.e., $\ell \equiv 23 \bmod 49$. In this case

$$
f_{\ell}(2) \equiv 0 \bmod 7^{2}, \quad f_{\ell}^{\prime}(2) \equiv 0 \bmod 7,
$$

so that Voronoi's theorem implies $u \equiv 4, v \equiv 3 \bmod 7$. This is in accordance with (2) modulo 49.

\section{Proof of the theorem}

For the basic facts concerning Voronoi's algorithm in totally real cubic fields, see [1], Chapter IV. For any number $\vartheta \in F_{\ell}$, let $\vartheta, \vartheta^{\prime}, \vartheta^{\prime \prime}$ (or $\vartheta^{(i)}, i=$ $0,1,2)$ be the conjugates, and let $\bar{\vartheta}=\left(\vartheta, \vartheta^{\prime}, \vartheta^{\prime \prime}\right)$ be the corresponding vector in $\mathbb{R}^{3}$. We choose the order of the conjugates so that

$$
1<\varepsilon<1+\ell^{-1}, \quad-\ell^{-1}<\varepsilon^{\prime}<0, \quad-\ell<\varepsilon^{\prime \prime}<-\ell+\ell^{-2} .
$$

Let $\Lambda=\{\bar{\vartheta} \mid \vartheta \in \mathcal{O}\}$ be the lattice in $\mathbb{R}^{3}$ corresponding to $\mathcal{O}$. The theorem is an immediate consequence of the following

Lemma 3. Suppose that $j \leq \ell / 3$. Let $\bar{\xi}$ and $\bar{\eta}$ be the relative minima of $\Lambda$ adjacent to $\overline{1}$ on the positive $x$ - and $y$-axis, respectively. Then

$$
\xi=(\varepsilon-1)^{-1}, \quad \eta=\varepsilon^{-1} .
$$

Proof. We apply the result of Lemma 2. Since $\varepsilon-1$ is a unit, it is clear that $\overline{(\varepsilon-1)^{-1}}$ is a relative minimum of $\Lambda$. It follows from (8) that

$$
\left|\varepsilon^{\prime}-1\right|^{-1}<1, \quad\left|\varepsilon^{\prime \prime}-1\right|^{-1}<1,
$$

so that $\xi$ must satisfy the conditions

$$
|\xi| \leq|\varepsilon-1|^{-1}, \quad\left|\xi^{\prime}\right|<1, \quad\left|\xi^{\prime \prime}\right|<1 .
$$

Since $\xi \in \mathcal{O}$, there are integers $x, y, z$ such that

$$
x+y \varepsilon^{(i)}+z \alpha^{(i)}=\xi^{(i)} \quad(i=0,1,2) .
$$

Consider (10) as a system of linear equations in the unknowns $x, y, z$. The determinant of the system is $-\sqrt{D} / j$. Here the square root is positive, and to get the correct sign we use (8). It follows that

$$
\sqrt{D} z / j=\left(\varepsilon^{\prime}-\varepsilon^{\prime \prime}\right) \xi+\left(\varepsilon^{\prime \prime}-\varepsilon\right) \xi^{\prime}+\left(\varepsilon-\varepsilon^{\prime}\right) \xi^{\prime \prime} .
$$

Changing the sign of $\xi$, if necessary, we may assume that $z \geq 0$. 
From (1) we have $\sqrt{D}>\ell^{2}+3 \ell-2$, so that (11), (9) and (8) imply

$$
\begin{aligned}
\left(\ell^{2}+3 \ell-2\right) z / j & <\left(\varepsilon^{\prime}-\varepsilon^{\prime \prime}\right) /(\varepsilon-1)+\varepsilon-\varepsilon^{\prime \prime}+\varepsilon-\varepsilon^{\prime} \\
& =\ell-1+3 \varepsilon+\varepsilon(\ell+\varepsilon)\left(\varepsilon^{\prime}-\varepsilon^{\prime \prime}\right) \\
& <\ell^{2}+3 \ell+6 .
\end{aligned}
$$

Since $j \leq \ell / 3$, it follows that $z \leq j$.

Subtract the equations (10) with $i=1,2$. Substituting the expressions of $\alpha^{\prime}$ and $\alpha^{\prime \prime}$ we obtain after a short computation

$$
j y-(\ell-v) z=(\varepsilon-1) z+j\left(\xi^{\prime}-\xi^{\prime \prime}\right) /\left(\varepsilon^{\prime}-\varepsilon^{\prime \prime}\right) .
$$

The absolute value of the right-hand side is less than

$$
j\left(\varepsilon-1+2 /\left(\varepsilon^{\prime}-\varepsilon^{\prime \prime}\right)\right) .
$$

We shall show that this expression is less than $3 j / \ell \leq 1$, so that the final result will be

$$
j y=(\ell-v) z \text {. }
$$

For that purpose it is enough to show that

$$
\left(1+3 \ell^{-1}-\varepsilon\right)\left(\varepsilon^{\prime}-\varepsilon^{\prime \prime}\right)>2 .
$$

The following improved bound for $\varepsilon$ is valid: $\varepsilon<r$, where

$$
r=1+\ell^{-1}-2 \ell^{-2}+4 \ell^{-3} \text {. }
$$

To see this, check that $f_{\ell}(1)<0, f_{\ell}(r)>0$. We then have

$$
\left(1+3 \ell^{-1}-\varepsilon\right)\left(\varepsilon^{\prime}-\varepsilon^{\prime \prime}\right)>\left(1+3 \ell^{-1}-r\right)\left(-\ell^{-1}+\ell-\ell^{-2}\right),
$$

which is easily seen to be $>2$ if $\ell \geq 4$. For $\ell=3$ one can simply compute the approximate values of $\varepsilon, \varepsilon^{\prime}, \varepsilon^{\prime \prime}$ and check that (14) holds even then.

We have thus proved (13). We contend that $(j, \ell-v)=1$ which implies

$$
y=\ell-v, \quad z=j,
$$

because $z \leq j, v<j \leq \ell / 3$, and the possibility $z=y=0$ is absurd.

Suppose that $\ell-v$ and $j$ are both divisible by a prime $p$. If $p=7$, then Lemma 1 gives $\ell \equiv 23 \bmod 49$, but then $v \equiv \ell \equiv 2 \bmod 7$ leads to a contradiction with (2). Hence $p \neq 7$. From (2) we have $h(\ell) \equiv 0 \bmod p$, where

$$
h(\ell)=\ell^{3}+\ell^{2}-19 \ell-30 .
$$

On the other hand

$$
\left(3 \ell^{2}+\ell-7\right) h(\ell)-(3 \ell-14) D=112(\ell-2),
$$

so that $\ell \equiv 2 \bmod p$ and $h(\ell) \equiv h(2) \equiv-56 \bmod p$, which is impossible.

Hence (15) is true, whence $\xi=x+u+\ell \varepsilon+\varepsilon^{2}=x+u+(\varepsilon-1)^{-1}$. Looking at $(8)$, one can see that the two last conditions (9) are only satisfied for 
$x+u=0$ or 1 . However, the first condition (9) does not hold for $x+u=1$. It follows that $\xi=(\varepsilon-1)^{-1}$, and the first part of the lemma is proved.

The proof that $\eta=\varepsilon^{-1}$ is very much the same. The number $\eta$ satisfies the conditions

$$
|\eta|<1, \quad\left|\eta^{\prime}\right| \leq\left|\varepsilon^{\prime}\right|^{-1}, \quad\left|\eta^{\prime \prime}\right|<1 .
$$

Again there are integers $x, y, z$ such that

$$
x+y \varepsilon^{(i)}+z \alpha^{(i)}=\eta^{(i)} \quad(i=0,1,2) .
$$

We may assume that $z \geq 0$, and we can prove that $z \leq j$ as before. Subtracting the equations (17) with $i=0,2$ we obtain the following analogue of (12):

$$
j y-(\ell-v-1) z=\varepsilon^{\prime} z+j\left(\eta-\eta^{\prime \prime}\right) /\left(\varepsilon-\varepsilon^{\prime \prime}\right) .
$$

In order to achieve the result

$$
j y=(\ell-v-1) z
$$

we have to show that the absolute value of the right-hand side of (18) is less than 1. This is true if we can show that

$$
\left(\varepsilon-\varepsilon^{\prime \prime}\right)\left(3 \ell^{-1}+\varepsilon^{\prime}\right)>2 .
$$

But (20) follows easily from (8). Hence (19) holds.

Suppose now that $j$ and $\ell-v-1$ are both divisible by a prime $p$. If $p=7$, then $\ell \equiv 23 \bmod 49$ and $v \equiv \ell-1 \equiv 1 \bmod 7$, which contradicts (2). Thus $p \neq 7$. Since $v \equiv \ell-1 \bmod p$, it follows from $(2)$ that $h(\ell)+28 \equiv 0 \bmod p$. On the other hand,

$$
\left(2 \ell^{3}+9 \ell^{2}+11 \ell-11\right)(h(\ell)+28)-\left(2 \ell^{2}-\ell-26\right) D=-784,
$$

which is impossible. As before, we now have

$$
y=\ell-v-1, \quad z=j,
$$

so that $\eta=x+u+\ell+\varepsilon^{-1}$. However, the first and third condition (16) are only satisfied for $x+u+\ell=0$. This completes the proof. 


\section{References}

[1] B. N. Delone, D. K. FAddeev, The Theory of Irrationalities of the Third Degree. Trudy Mat. Inst. Steklov, vol. 11 (1940); English transl., Transl. Math. Monographs, vol. 10, Amer. Math. Soc., Providence, R. I., Second printing 1978.

[2] V. EnNola, Cubic number fields with exceptional units. Computational Number Theory (A. Pethö et al., eds.), de Gruyter, Berlin, 1991, pp. 103-128.

[3] H. G. Grundman, Systems of fundamental units in cubic orders. J. Number Theory 50 (1995), 119-127.

[4] M. Mignotte, N. Tzanakis, On a family of cubics. J. Number Theory 39 (1991), 41-49, Corrigendum and addendum, 41 (1992), 128.

[5] E. THOMAS, Fundamental units for orders in certain cubic number fields. J. Reine Angew. Math. 310 (1979), 33-55.

Veikko ENNOLA

Department of Mathematics

University of Turku

FIN-20014, Finland

E-mail : ennola@utu.fi 